\title{
Developing a modern thermal strengthening technique for regulated fishplate cooling
}

\author{
Y. G. Yaroshenko ${ }^{1}$, Y. I. Lipunov ${ }^{2}$, M. V. Startseva ${ }^{1}$, \\ K. Y. Eysmondt ${ }^{2}$, E. V. Nekrasova ${ }^{2}$ \& G. G. Trayanov ${ }^{2}$ \\ ${ }^{I}$ Department of Metallurgy, Ural Federal University, Russian Federation \\ ${ }^{2}$ The Centre of Advanced Cooling Systems, \\ Scientific-Research Institute of Metallurgical Heat Engineering - OJSC \\ "VNIIMT", Russian Federation
}

\begin{abstract}
A modern ecologically friendly technique for fishplate (joint bar) thermal strengthening by regulated water cooling is proposed. The proposed technique can successfully replace the conventional one - oil quenching - due to making the cooling process more controlled by obtaining a uniform temperature distribution over fishplate elements with a different mass such as heads and the wet. The oil quenching process is an out of control fire hazardous one and requires controlling the permanent cooling capability, fishplate flushing after the operation. It is necessary to utilize the oil and to have a special ventilation system. As a mineral oil substitute, the usage of polymer media has been investigated. Within the time, the polymer solution needs to be recycled; the high cost of polymer concentrates also plays an important role. Quenching by water spraying is the most ecological and controlled type of thermal strengthening. The technique was validated by a test bench experiment and implemented in the metallurgical industry. The mechanical properties have been obtained to meet the GOST 4133-73 technical requirements. Moreover, the analysis made showed that when the controlled cooling device is fitted into the line existing due to exclusion of the hardening tank and washing machine from the processing flow it is possible to save energy, exclude the expenditures for acquisition, the preparation of oil and washing mixtures as well as for regeneration of the oils used and washing solutions.
\end{abstract}

Keywords: joint bar (fishplate), accelerated water cooling, thermohardening of rolling metal, sprayer systems. 


\section{Introduction}

A fishplate is one of the major elements of the permanent way when rails are jointed end to end and permits to extend the wear-life of a continuous welded rail (CWR) (Ivanov and Dankov [1]). Quenching being an ending operation of the fishplate production influences its microstructure and mechanical properties. It can be done in different quenchants, namely water, polymers, and oils and, depends upon technology requirements as to the rate not less than the critical cooling rate for particular kind of rolling metal (Yao Ji-Hoon [2]). Bramfitt et al. [3] showed that oil-quenched fishplate can be replaced with microalloyed one but it requires quite significant amount of microalloyed elements such as manganese, vanadium, aluminum. In accordance with the GOST 4133-73 technical requirements thermal strengthening of fishplates should be done by immersion quenching in the mineral oil bath. This process is an out of control fire hazardous one and requires controlling permanent cooling capability, fishplate flushing after the operation. It is necessary to utilize the oil and to have a special ventilation system. As a mineral oil substitute, the usage of polymer media has been investigated (such as iron-containing polyacrylic acid salt PC-2 and sodium salts of carboxymethylcellulose $\mathrm{Na}-\mathrm{CMC}$ ) (Zakharov et al. [4]). It is possible to improve the fishplate performance by reducing the pearlite interlamellar distance and the amount of the free ferrite structure in the metal surface layer as it has been confirmed by investigations at a metallurgical plant by quenching a fishplate in an aqueous solution PC-2 (Pavlov et al. [5]). Good results of the mechanical properties with respect to polymer PC-2 certain concentrations were obtained and the relation equation of heat-transfer coefficient $\alpha$, the initial PC-2 concentration and the temperature of the bath at various surface temperatures of products were derived (Zakharov et al. [4]). Eysmondt et al. [6] found that the change of PC-2 cooling properties is shown depending on time: having experienced more than 100 quenching the solution completely changed its cooling capacity for the worse because the duration of the film boiling stage had been increased. It can be restored by adding a new portion of solution, but the frequency and amount of recharging should be determined only empirically. Despite of all the advantages of the polymer medium, the immersion quenching cannot permit to organize different cooling conditions for the complex asymmetrical cross-section fishplate, while the need for careful monitoring of polymer concentration, its cooling capacity, the temperature and other parameters remain. Within the time, the solution needs to be recycled; the high cost of polymer concentrates also plays the important role. Quenching by water spraying is the most ecological and controlled type of thermal strengthening. The quenching technique by a water sprayer system has not been examined for fishplates. Therefore, the objective of the present paper is to investigate the possibility of this process. 


\section{Numerical simulation of the process}

At the first step of the proposed technique implementation, the numerical simulation of the process were performed using a previously developed numerical model based on the solution of the two-dimensional Fourier heat eqn (1) taking into account that thermophysic properties of steel depend on the temperature:

$$
\rho c(t) \frac{\partial t}{\partial \tau}=\frac{\partial}{\partial x}\left[\lambda(t) \frac{\partial t}{\partial x}\right]+\frac{\partial}{\partial y}\left[\lambda(t) \frac{\partial t}{\partial y}\right]
$$

where $t$ is temperature, $\mathrm{K} ; c, \rho, \lambda$ are the material's heating capacity, density and conductivity respectively; $x, y$ are coordinates of the fishplate's crosssection, $\tau$ is time, sec.

The following conditions are used:

a) the initial condition:

$$
t\left(\tau_{0}\right)=t_{\text {init. }},
$$

where $t_{\text {init. }}$ is the initial temperature distribution over the cross-section of the workpiece, $\mathrm{K}$.

b) cooling by free convection:

$$
\left\{\begin{array}{l}
\left.\lambda(t) \frac{\partial t}{\partial x}\right|_{x=0}=\alpha(t)\left(t_{\text {surf }}-t_{\text {sur.med. }}\right)+\varepsilon_{M} c_{0}\left[\left(\frac{T_{\text {surf }}}{100}\right)^{4}-\left(\frac{T_{\text {sur.med. }}}{100}\right)^{4}\right] \\
\left.\lambda(t) \frac{\partial t}{\partial y}\right|_{y=0}=\alpha(t)\left(t_{\text {surf }}-t_{\text {sur.med. }}\right)+\varepsilon_{M} c_{0}\left[\left(\frac{T_{\text {surf }}}{100}\right)^{4}-\left(\frac{\left.T_{\text {sur.med. }}\right)^{4}}{100}\right],\right.
\end{array}\right.
$$

where $\alpha$ is the heat-transfer coefficient, $\mathrm{W} /\left(\mathrm{K} \cdot \mathrm{m}^{2}\right), \varepsilon_{m}$ is the emissivity factor of the surface, $\varepsilon_{m} \approx 0.8 \div 0.85 ; c_{0}$ is the radiation coefficient of a black body $5.67 \mathrm{~W} / \mathrm{M}^{2} \mathrm{~K}^{4} ; t_{\text {surf }}, T_{\text {surf }}$ are the surface temperature, $\mathrm{K} ; t_{\text {sur.med. }}$. $T_{\text {sur.med. }}$ are the surrounding medium temperature, $\mathrm{K}$.

c) cooling by water jets:

$$
\left\{\begin{array}{l}
\left.\lambda \frac{\partial t}{\partial x}\right|_{x=0}=\left\{\begin{array}{l}
q \text { if } t_{\text {surf }} \geq 100^{\circ} C \\
\alpha(t)\left(t_{\text {surf }}-t_{\text {sur.med. }}\right)
\end{array}\right) \\
\left.\lambda \frac{\partial t}{\partial y}\right|_{y=0}=\left\{\begin{array}{l}
q \text { if } t_{\text {surf }} \geq 100^{\circ} C \\
\alpha(t)\left(t_{\text {surf }}-t_{\text {sur.med. }}\right)
\end{array}\right)
\end{array}\right.
$$

where $q$ is the heat-flux density at the surface, $\mathrm{MW} / \mathrm{m}^{2}$.

The heat-flux density for boundary conditions eqn (4) was determined by using experimental curves obtained using industrial water sprayer systems (Eysmondt [7]). The main constructive sprayer system characteristics and preliminary thermal strengthening modes were determined based on the obtained 
temperature versus time change relation for different cross-section points of the fishplate.

\section{Experimental work and test bench results}

According to the results of the numerical simulation, a sprayer system consisting of two sections was proposed; there was an air gap between sections. Each section, in turn, was composed of several cooling contours (Fig. 1), a crosssectional view of one of them is shown in Fig. 1(b). A contour represents a system of flat water jets intersecting in the same vertical plane and completely covering the surface of the fishplate. Each delivery pipeline independently provides the possibility of establishing different conditions for cooling the wet and heads, as well as an arrangement of nozzles at the right angle to the surface clearly restricts the cooling zone and the air gap in between. All of these steps allowed obtaining a uniform temperature field over the cross-section fishplates, thus, ensuring the GOST technical requirements and controlling the cooling process.
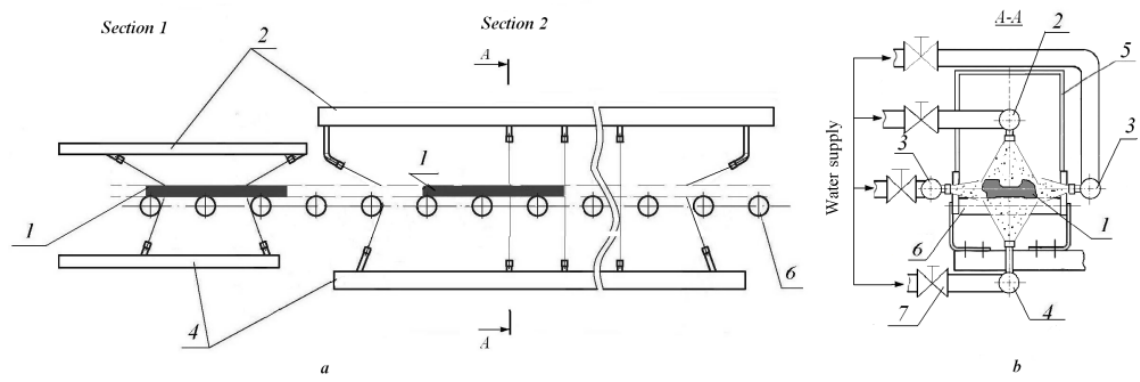

Figure 1: Scheme of the regulated cooling sprayer system. (a) side-view of the system; (b) cross-sectional view of a contour. (1 - R65 type fishplate; 2 - top delivery pipeline with the nozzles; 3 - side delivery pipelines with the nozzles; 4 - bottom delivery pipeline with the nozzles; 5 - casing; 6 - roller; 7 - faucet.)

The proposed design of the system was mounted at the test bench, and the second step of conducting a series of experimental investigation was implemented. The requirements to the heating and holding process were taken into consideration, i.e. the specimen was heated in gas furnace till austenitizing temperature $870-880^{\circ} \mathrm{C}$. The heated specimen was cooled in the system, continuously recording the surface temperature before, during and after the process. To realize it, chromel-alumel thermocouples were welded on the surface of the wet's fishplate, and data was recorded by a measuring set with the time frequency of 0.1 seconds.

The set consists of chromel-alumel thermocouples, compensating cables, an input/output module and an analog to digital signals converter and PC, where measurement data was automatically recorded into the memory. An error of the 
set was $\pm 7^{\circ} \mathrm{C}$. The data of the thermocouple in one of the experiments is shown in Fig. 2. In order to further monitor the temperature after the process, two kinds of pyrometers were applied: a stationary pyrometer "Thermoscope-800" and a hand pyrometer "Thermoscope-100". Fig. 3 shows the temperature distribution along the length of the fishplate, measured of the hand pyrometer at the exit of the system and the furnace. It can be seen that the system design chosen and process parameters achieved a uniform temperature distribution for the elements with different mass and along the fishplate length.

Thermal experiments helped to clarify the constructive parameters of the system and to determine the thermal modes where the uniform temperature field for the wet and was achieved. Technological experiments allowed to investigate the microstructure obtained and to perform a complex mechanical test by using specified thermal modes. Mechanical properties of R65 type fishplates (one of the most widespread type) in the series of experiments and the ones in accordance with GOST 4133-73 technical requirements are shown in Table 1.

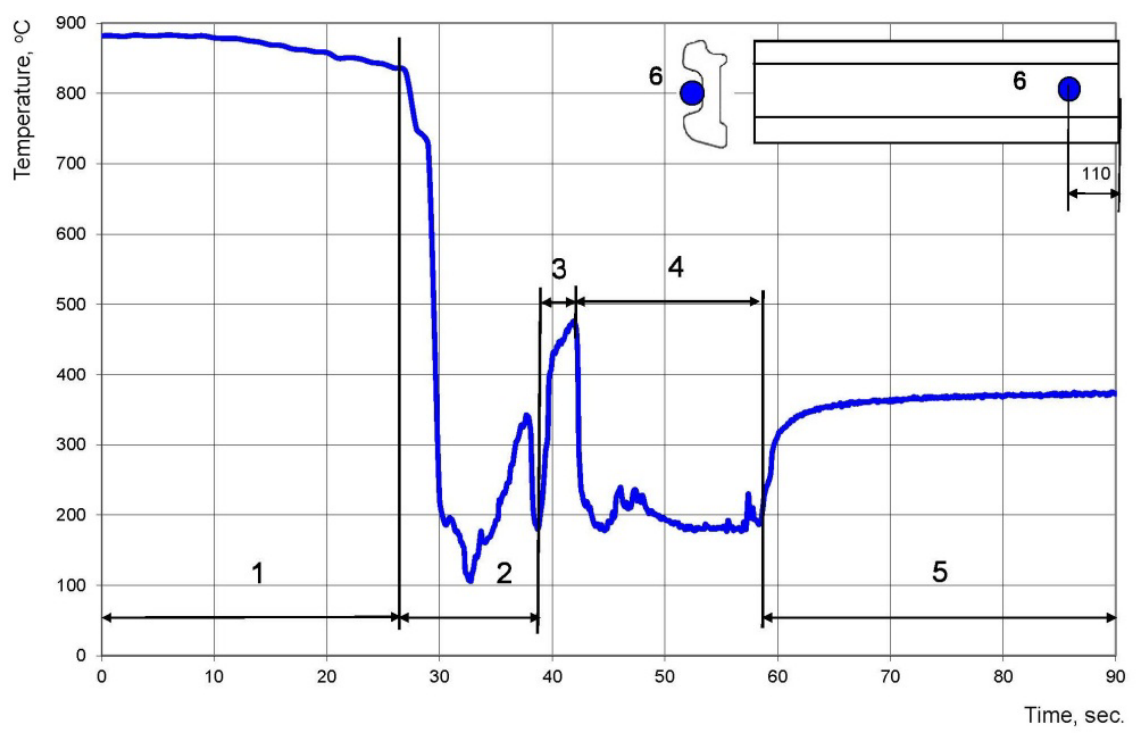

Figure 2: Changes of the temperature during the process of controlled water cooling system (experiment № 2 at 10.02.2012). (1 - specimen air cooling released from furnace; 2 - cooling in section № $1 ; 3$ - the change of the surface temperature at the air gap between sections № 1 and № 2; 4 - cooling in section № 2, 5 - the change of the surface temperature when cooling is over, $6-$ the point where chromel-alumel thermocouple was established, the surface center of the wet.) 


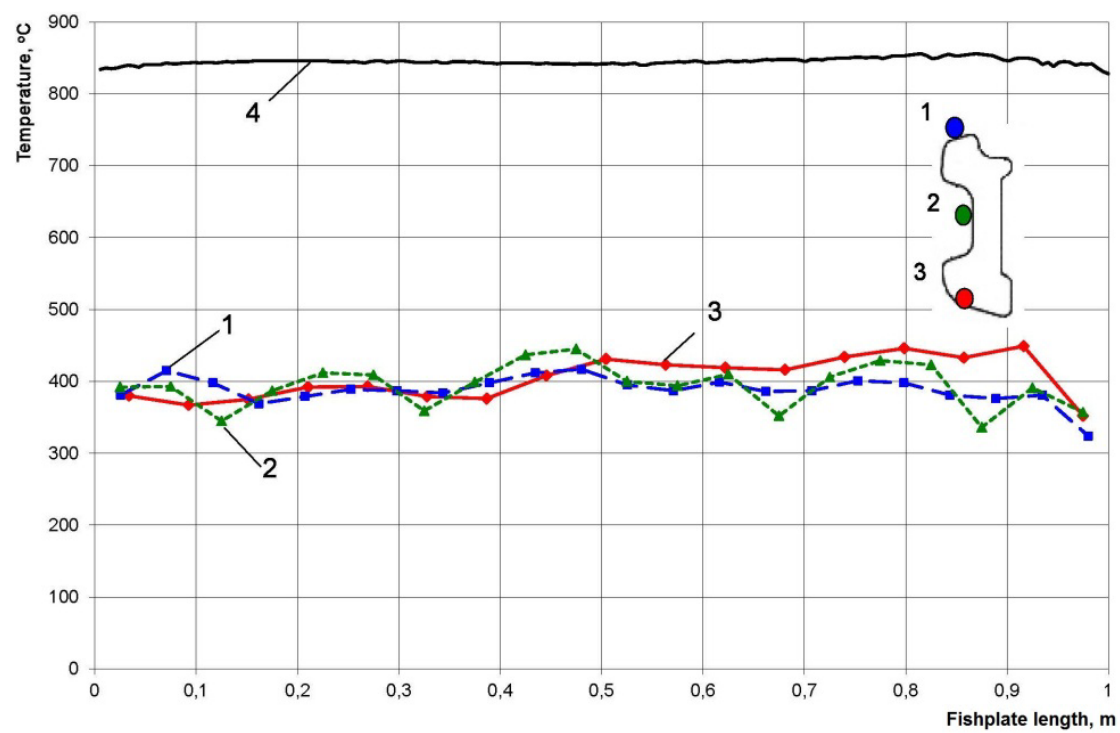

Figure 3: The temperature distribution along the length of the fishplate after thermal strengthening $(30 \mathrm{sec}$.), the data of the bench experiment № 3 on February, 27, 2012. (1 - top head, 2 - wet, 3 - bottom head, 4 - the wet temperature at the outlet of the furnace.)

Table 1: Mechanical properties of R65 type fishplates (6-bolted specimen, the length $1000 \mathrm{~mm}$ ).

\begin{tabular}{|c|c|c|c|c|c|c|}
\hline Fishplate & $\begin{array}{l}\text { Tensile } \\
\text { strength, } \\
\mathrm{MH} / \mathrm{M}^{2}\end{array}$ & $\begin{array}{l}\text { Yield } \\
\text { stress, } \\
\mathrm{MH} / \mathrm{M}^{2}\end{array}$ & $\begin{array}{c}\text { Tensile } \\
\text { strain, } \\
\%\end{array}$ & $\begin{array}{c}\text { Reduction } \\
\text { of area, } \\
\%\end{array}$ & $\begin{array}{l}\text { Bending } \\
\text { angle, } \\
\text { degree }\end{array}$ & $\begin{array}{c}\text { Brinell } \\
\text { hardness } \\
\text { HB } \\
\text { (in limits) }\end{array}$ \\
\hline $\begin{array}{l}\text { Test bench } \\
\text { experiments }\end{array}$ & $\begin{array}{l}893 \div \\
1012\end{array}$ & $\begin{array}{c}570 \div \\
694\end{array}$ & $\begin{array}{c}10.4 \div \\
17.4\end{array}$ & $\begin{array}{c}41.6 \div \\
49.3\end{array}$ & $\begin{array}{l}46 \div \\
106\end{array}$ & $285 \div 363$ \\
\hline $\begin{array}{c}\text { Technical } \\
\text { requirement } \\
\text { "GOST 4133- } \\
\text { 73", more than }\end{array}$ & 844 & 530 & 10 & 30 & 20 & $235 \div 388$ \\
\hline
\end{tabular}

\section{Experimental work and industrial results}

\subsection{Mechanical properties investigation}

The system of regulated cooling is implemented in the company branch "EVRAZ NTMK" NSMZ. Rolling metal is used in two varieties differing in carbon content: "EVRAZ ZSMK" and "EVRAZ NTMK". TC 14-2R-463-2011 specifications for this plant were developed using as the basis of the GOST 
4133-73 technical requirements. The thermal mode was identified for each rolling metal sort based on the data of bench investigation and mechanical properties for the corresponding modes. The results are shown in Table 2 .

Table 2: Mechanical properties of R65 type fishplates with different carbon content thermal strengthening at "EVRAZ NTMK" NSMZ.

\begin{tabular}{|c|c|c|c|c|c|}
\hline Fishplates & $\begin{array}{c}\text { Tensile } \\
\text { strength, } \\
\mathrm{MH} / \mathrm{M}^{2}\end{array}$ & $\begin{array}{c}\text { Yield } \\
\text { stress, } \\
\mathrm{MH} / \mathrm{M}^{2}\end{array}$ & $\begin{array}{c}\text { Tensile } \\
\text { strain, } \\
\%\end{array}$ & $\begin{array}{c}\text { Reduction } \\
\text { of area, } \\
\%\end{array}$ & $\begin{array}{c}\text { Brinell } \\
\text { Hardness } \\
\text { HB } \\
\text { (in limits) }\end{array}$ \\
\hline $\begin{array}{c}\text { «NTMK» } \\
\text { production } \\
0.49-0.51 \% \mathrm{C}\end{array}$ & $963-985$ & $640-655$ & $12-16$ & $31-41$ & $236-269$ \\
\hline $\begin{array}{c}\text { «MK» } \\
\text { production } \\
0.56-0.58 \% \mathrm{C}\end{array}$ & $872-912$ & $545-555$ & $13-19$ & $31-37$ & $241-255$ \\
\hline $\begin{array}{c}\text { Technical } \\
\text { requirement } \\
\text { "TC } 14-2 \mathrm{P}- \\
\begin{array}{c}463-2011 ", \\
0.45-0.62 \% \mathrm{C}\end{array}\end{array}$ & $\begin{array}{c}\text { more than } \\
844\end{array}$ & $\begin{array}{c}\text { more than } \\
530\end{array}$ & $\begin{array}{c}\text { more than } \\
10\end{array}$ & $\begin{array}{c}\text { more than } \\
30\end{array}$ & $\begin{array}{c}\text { In limits } \\
235-388\end{array}$ \\
\hline
\end{tabular}

\subsection{Microstructure investigation}

The microstructure of the bottom head before and after application of the bench experiment, using an optical microscope is shown in Fig. 4.

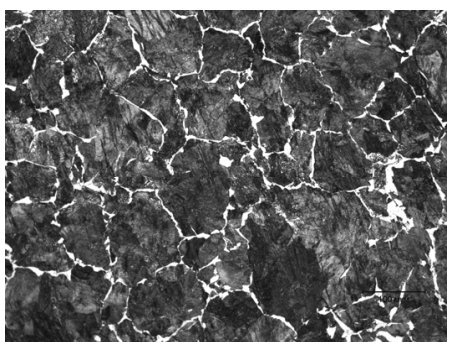

(a)

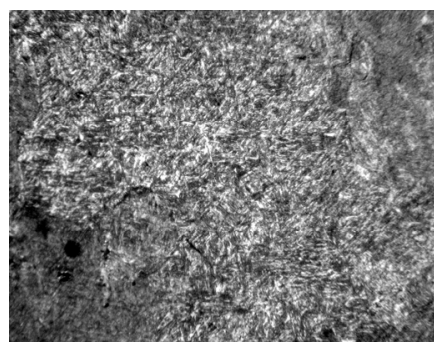

(b)

Figure 4: The microstructure of the fishplate bottom head before (a) and after thermal strengthening (b) (x200).

The initial structure (before heat treatment, Fig. 4(a)) consists of lamellar pearlite and ferrite oriented along the grain boundaries. The microstructure after heat treatment (Fig. 4(b)) is denser perlite with a strong ferrite grain boundary on the grid. The investigation of the structure after thermohardening with a higher 
degree of increase was carried out using SEM JEOL JSM-6490LV. Figure 5 shows the structure of the central part of the top head (a) and at 500 microns from the edge (b).

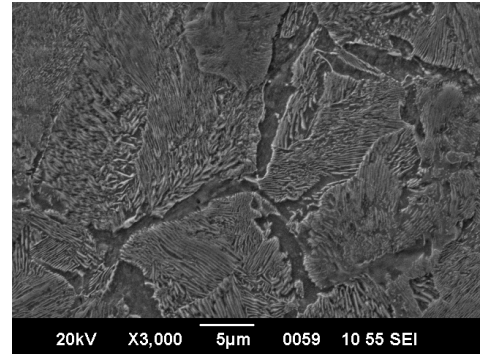

(a)

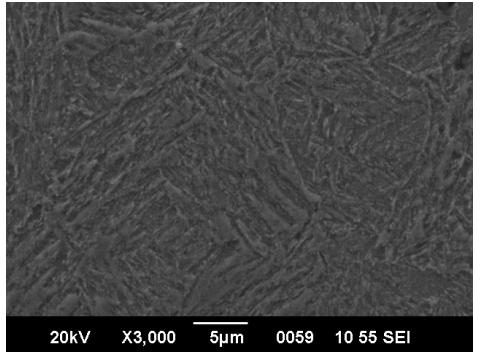

(b)

Figure 5: The microstructure of the middle part top head (a) and at a distance of $500 \mathrm{~mm}$ from the upper edge of the head (b) (x3000).

The structure of the middle part as is seen in the Fig. 5 consists of the perlite and ferrite at a grain boundary, the grain size of approximately $10-15$ microns, surface layer structure is secondary sorbite. Thus, in the thermal strengthening process, the metal grain size and pearlite lamellar spacing were reduced.

\section{Conclusion}

As a result of the test bench research and numerical modeling, a new thermal strengthening process has been tested, design features of the controlled cooling system and heating modes were defined. Mechanical properties of the fishplate meet technical requirements and are not inferior to mechanical properties of the fishplates oil immersion quenched. The system allows us to obtain a uniform temperature distribution for the elements with different mass and, hence, ensures warpage requirements. Environmentally friendly technology has indisputable advantages over the conventional quenching due to using the water circulating in a closed loop. Currently, the method is being implemented in industry.

\section{Acknowledgements}

The authors would like to acknowledge the support provided by the following organizations: Scientific-Research Institute of metallurgical Heat Engineering VNIIMT, EVRAZ NTMK - NSMZ, Ural Federal University named after the first President of Russia B. N. Yeltsin, Hong Kong University of Science and Technology. 


\section{References}

[1] Ivanov P.S., Dankov S.F., The new resource-saving techniques in the railway sector, Railroad and track facilities, 7, pp.23-25, 2011.

[2] Yao Ji-Hoon, Cooling and choice of media in steel quenching process, Induction heating, 18, pp.9-16, 2011.

[3] Bramfitt, B.L., Hansen, S.S., Wirick, D.P., Collins, W.B., Development of a microalloyed joint bar, Proc. Microalloying'88 and World Mater. Congr., vol. 8, eds. S.G. Fishman \& A.K. Dhingra, ASM: Chicago, pp.451-457, 1988.

[4] Zakharov, A.V., Eysmondt, Y.G., Shcherbakova, L.P., Investigation of the $\mathrm{Na}-\mathrm{CMC}$ polymeric medium usage possibility for rails and rail fastening quenching, Metal Science and Heat Treatment, 4, pp.24-27, 1991.

[5] Pavlov, V.V., Korneva, L.V., Pyataikin, E.M., Kozirev, N.A., et al. A technique of fishplate thermal strengthening, Patent RU 2291206 C1 2005. Federal Institute of Industrial property, http://www1.fips.ru/wps/wcm /connect/content_en/en/Informational_resources/Retrieval_System/.

[6] Eysmondt, Y.G., Gervasiev, M.A., Trunina, T.A., Investigation of the fishplate's quenching possibility in a polymeric medium, Steel in translation, 9, pp.71-73, 2000.

[7] Eysmondt, K.Y., Development and implementation in industry of thermal strengthening system for rolled metal controlled cooling using as the basis the heat transfer analysis, Ph.D. thesis, USTU: Yekaterinburg, p.170, 2011. 